\title{
Variation in CD4 cell count among IDUs versus sexually-infected HIV-positive naïve patients in Romania
}

\author{
Iulia Niculescu*, Eliza Manea1', Simona Paraschiv², Ionelia Bâțan², Adrian Abagiu', Adriana Hristea ,3, Raluca Jipa', \\ Cătălin Tilişcan ${ }^{1,3}$, Raluca Mihăilescu' ${ }^{1}$, Adrian Streinu-Cercel ${ }^{1,3}$, Victoria Aramă ${ }^{1,3}$, Dan Oțelea ${ }^{2}$ \\ From The 7th Romanian National HIV/AIDS Congress and The 2nd Central European HIV Forum \\ Sibiu, Romania. 29-31 May 2014
}

Since 2011 Romania is experiencing a dramatic increase of newly diagnosed HIV infections among injecting drug users (IDUs), mainly linked to the introduction of new psychoactive substances (NPS) on the Romanian market, their use being related to higher levels of risk behavior compared to opioid abuse. There is no sufficient data showing the natural course of HIV in subjects infected through IDU - the majority are ART-naïve due to recently acquired infection (many are asymptomatic and have a CD4 cell count over 350 cells/cmm) and poor adherence. Our objective was to determine if IDUs have a faster decline in CD4 cells than sexually-infected patients.

We performed a retrospective study, including ARTnaïve HIV-positive patients diagnosed between January 2011 and June 2013 at the National Institute for Infectious Diseases "Prof. Dr. Matei Balş", who had 2 subsequent CD4 cell count determinations over a timespan of 6-24 months and a baseline CD4 count higher than 350 cells/ $\mathrm{cmm}$. Among 1,248 newly diagnosed patients, $234 \mathrm{met}$ these inclusion criteria. The data were statistically analyzed using SPSS version 17 (independent sample t-test, MannWhitney test, linear regression; the significance level was set at 0.05 ).

The majority $(80 \%, 187 / 234)$ were men and the median age was 29 years (15-76). More than half of the patients $(138 ; 59 \%)$ were former or active IDUs, with low socioeconomic status, most of them injecting both opioids and NPS and 55 (40\%) of them were in detention at the moment of HIV diagnosis. Among them, $98 \%$ were coinfected with HCV, as opposed to only $21 \%$ of the sexually-infected patients. Subtype analysis was performed for 64 patients and revealed the following subtypes and circulating recombinant forms (CRF): 50 F1, 3 B, 10 CRF14_BG and 1 CRF14_F. There was no significant difference of $\mathrm{CD} 4$ cell count between the two groups at baseline $(\mathrm{p}=0.55)$. The median variation of CD4 cells in IDUs was 150 and 42 in the non IDUs group. IDUs had a statistically significant CD4 cell decline $(\mathrm{p}=0.01)$. HCV coinfection was also correlated with a faster decline in CD4 cells ( $\mathrm{p}<0.001$ ).

The more rapid decline of CD4 cells among IDUs could be explained by direct effect of drugs, differences in the virulence of the HIV strains circulating among IDUs and HCV coinfection. These data highlight the importance of carefully monitoring IDUs with HIV infection and of initiating ART earlier in this risk group.

\section{Authors' details}

${ }^{1}$ Clinical Department, National Institute for Infectious Diseases "Prof. Dr. Matei Balş", Bucharest, Romania. Molecular Diagnostics Laboratory, National Institute for Infectious Diseases "Prof. Dr. Matei Balş", Bucharest, Romania. ${ }^{3}$ Carol Davila University of Medicine and Pharmacy, Bucharest, Romania.

Published: 29 May 2014

doi:10.1186/1471-2334-14-S4-011

Cite this article as: Niculescu et al:: Variation in CD4 cell count among IDUs versus sexually-infected HIV-positive naïve patients in Romania. BMC Infectious Diseases 2014 14(Suppl 4):O11. 